\title{
Preface to the Focused Issue on Fractional Derivatives and General Nonlocal Models
}

\author{
Qiang $\mathrm{Du}^{1} \cdot$ Jan S. Hesthaven ${ }^{2} \cdot{\text { Changpin } \mathrm{Li}^{3} \cdot \text { Chi-Wang Shu }}^{4} \cdot$ Tao Tang $^{5}$ \\ Published online: 20 September 2019 \\ (c) Shanghai University 2019
}

Fractional calculus, which has two prominent characteristics - singularity and nonlocality, comprises the integration and differentiation of any positive real (and even complex) order. It has a more than three-hundred-year history and can be traced back to a letter from Leibniz to L'Hôpital, dated 30 September 1695, in which the meaning of the one-half order derivative was first discussed and some remarks about its feasibility were made. Abel was probably the first who rendered an application of fractional calculus. He used the derivatives of arbitrary order to solve the tautochrone (isochrone) problem in 1823. Fractional calculus underwent two periods: from its beginning to the 1970s, and after 1970s. During the first period, fractional calculus was studied mainly by mathematicians as an abstract field containing only pure mathematical manipulations of little applications, except for sporadic applications in rheology. During the second period, the paradigm shifted from pure mathematical research to applications in various realms, including anomalous diffusion, anomalous convection, power laws, allometric scaling laws, history dependence, longrange interactions, and so on.

It is known that the important feature of fractional models is nonlocality. In our more and more connected world, nonlocal interactions are becoming increasingly prominent. This has fueled the growth of studies on nonlocal modeling, analysis and computation.

Chi-Wang Shu

chi-wang_shu@brown.edu

Qiang Du

qd2125@columbia.edu

Jan S. Hesthaven

Jan.Hesthaven@epfl.ch

Changpin Li

lcp@shu.edu.cn

Tao Tang

tangt@sustc.edu.cn

1 Columbia University, New York, USA

2 Ecole Polytechnique Fédérale de Lausanne, Lausanne, Switzerland

3 Shanghai University, Shanghai, China

4 Brown University, Providence, USA

5 Southern University of Science and Technology, Shenzhen, China 
Indeed, one can find relevant works ranging from practical applications in anomalous diffusion, fracture mechanics, and social dynamics, to rigorous development of nonlocal vector calculus, nonlocal function spaces and nonlocal calculus of variations. A particular development in this area deals with nonlocal models with a finite range of nonlocal interactions, which also serve as bridges between conventional local models (the limit when the nonlocal interactions get localized) and fractional models (the limit of infinite range of interactions using special power-like functions as nonlocal interaction kernels).

The present focused issue entitled "fractional derivatives and general nonlocal models" in Communications on Applied Mathematics and Computation (CAMC) covers fractional derivatives problems and nonlocal problems. The first part is published this month, and the remaining will be published in the coming issues. We do hope this focused issue can offer fresh stimuli for the community of fractional calculus and the modeling of nonlocal problems to promote and develop cutting-edge research in this important field.

Focused Issue Editors

Qiang Du

Jan S. Hesthaven

Changpin Li

Chi-Wang Shu

Tao Tang 